\title{
THE INFLUENCE OF VARYING GINGIVAL DISPLAY OF MAXILLARY ANTERIOR TEETH ON THE PERCEPTIONS OF SMILE AESTHETICS.
}

\author{
J Sybaite, P Sharma, P Fine, R Blizard and A Leung \\ UCL Eastman Dental Institute
}

\section{Keywords:}

Smile design, Smile aesthetics, Gingival display, High smile line(s), Gingival margin(s), Gingiva anatomy

\begin{abstract}
Objectives

The aim of the study was to investigate the influence of gingival display on perceived smile aesthetics and to compare aesthetic gingival display preference amongst dental professionals and lay people.

\section{Methods}

This cross-sectional study was based on the analysis of quantitative data collected via questionnaires. Two sets of digitally altered smile images with variations of gingival amount and architecture displays were produced. Ranking order scale was used to record aesthetic preferences, from the most attractive to the least attractive.
\end{abstract}

\section{Results}

There were 124 respondents (60 lay people, 32 general dentists (GDPs) and 32 orthodontists). Average smile line, tangent to the zeniths of maxillary anterior teeth, was found to be the most attractive gingival amount $\left(53.2 \%, \chi^{2}=144.15, \mathrm{p}<0.045\right)$ amongst lay people, GDPs and orthodontists. High smile line was ranked as the least attractive amount amongst all respondents $\left(88.7 \%, \chi^{2}=359.37 \mathrm{p}<0.002\right)$. Class III gingival outline, where all gingival levels are symmetrical and in line, was found as the most attractive gingival architecture $\left(65.3 \%, \chi^{2}\right.$ $=10.87 \mathrm{p}<0.001)$.

\section{Conclusion}

No particular amount of gingival display is universally perceived as attractive although high smile lines are generally disliked. Class III gingival architecture is the most preferred gingival archetype when excessive amount of maxillary gingiva is exposed. 


\section{Clinical significance}

A general acceptance to smile line variations demonstrates that the harmony amongst all smile components is more important than merely focusing on a single ideal smile constituent. However, when designing the smile with an inherent high lip line, a Class III gingival architecture might be more preferable since it reduces the negative impact of excessive gingival display.

\section{INTRODUCTION}

Human social existence is based on the premise that "what is beautiful is good" (1). Visual perception of beauty is determined both by nature and nurture and ultimately is how individuals feel about what they see (2).

Beauty perception principles can also be applied to smile aesthetics. If perception of smile aesthetics is objective, it will be easy to develop a universally accepted ideal smile. On the contrary, what one individual perceives as beautiful might not be appealing to another. Likewise, what is attractive to a dental professional might not be attractive to a patient (3). It is therefore challenging to quantify and qualify a universal recipe for a beautiful smile. While aesthetics is traditionally attributed to the field of arts, scientific principles can be utilised to achieve optimum dental aesthetic outcomes (4).

Gingivae is an essential component of smile aesthetics that provides a frame around every tooth and is part of the smile design process, often referred to 'pink aesthetics' $(5,6)$. When smiling, upper and lower lips create a line extending from one corner of one lip to the other and is known as a smile line, or inter-commissural line. In a vertical dimension, smile line can be described as low, average or high in relation to upper maxillary teeth (7) (Figure 1). Low smile line reveals less than $75 \%$ of maxillary incisors with no maxillary gingival tissues visible; average smile reveals $75-100 \%$ of incisors and interproximal papillae only; high smile exposes $100 \%$ of incisors and a continuous band of maxillary gingiva.

Excessive gingival display (EGD) is commonly known as a "gummy smile" and is described as overexposure of maxillary gingival band during voluntary or involuntary smiling (8). The clinical research of maxillary gingival display suggests that EGD of more than $3 \mathrm{~mm}$ is generally perceived as not aesthetic. Subsequently, smile design advocates the gingival display to be within a range of low to average smile line. This range is associated with a more youthful and attractive smile (9). A low smile line is considered to be a trait of aging and is not typically a desired outcome amongst patients (10). 


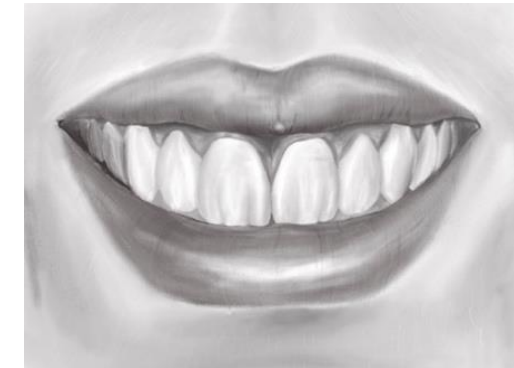

High Smile

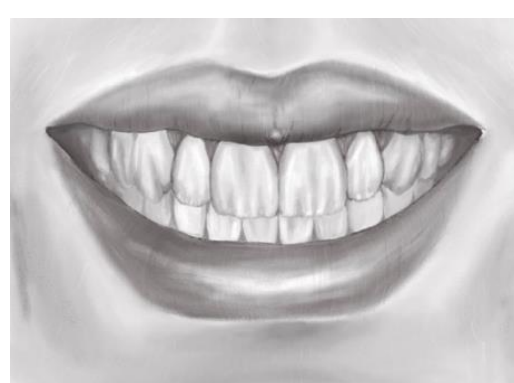

Average Smile

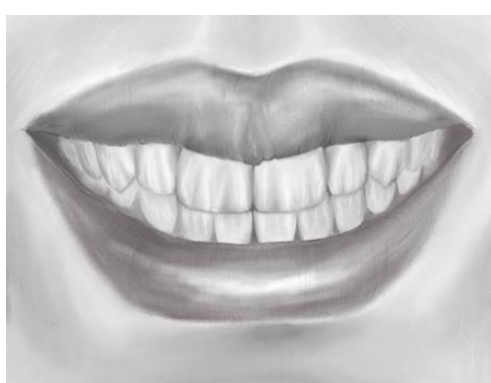

Low Smile

Figure 1. Smile line classification. (Illustrations in Figures 1-5 are drawn by one of the authors)

Progression of gingival margin levels from central incisor to canine constitutes an architecture of gingival contour (11) (Figures 2-5). A line drawn through the tangents of gingival zeniths of central incisor and canine is known as the gingival aesthetic line (GAL). Gingival architecture in relation to this line allows classification into four different groups as shown in Figures 2-5 (12) thus allowing to assess the influence of various archetypes to perceived smile aesthetics. As a general rule, bilateral symmetry and gingival architecture of Class I without black triangles are desirable aesthetic gingival parameters (13-15).

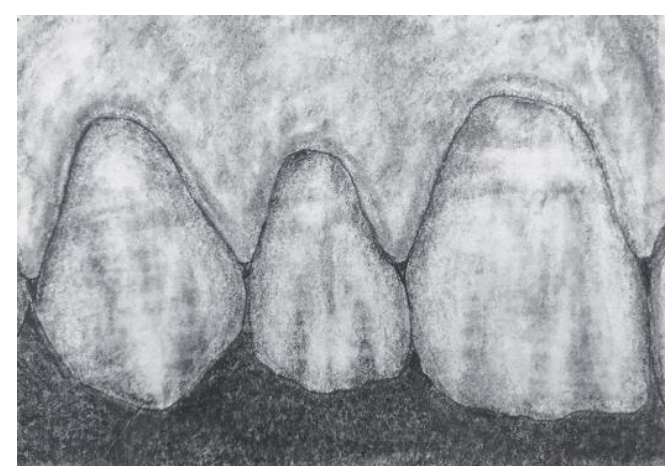

Figure 2. Class I. Lateral incisors below the GAL

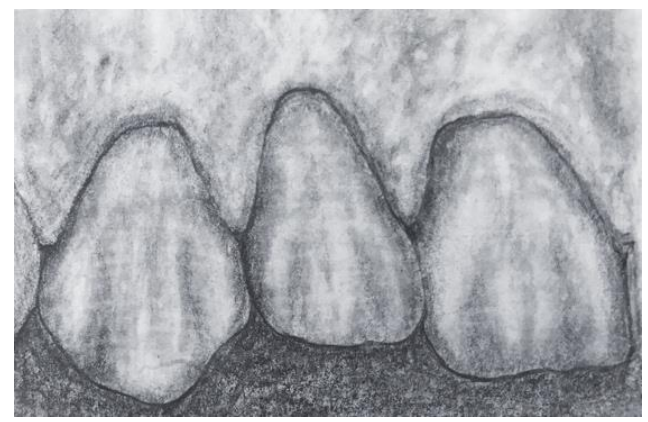

Figure 3. Class II. Lateral incisor above the GAL 


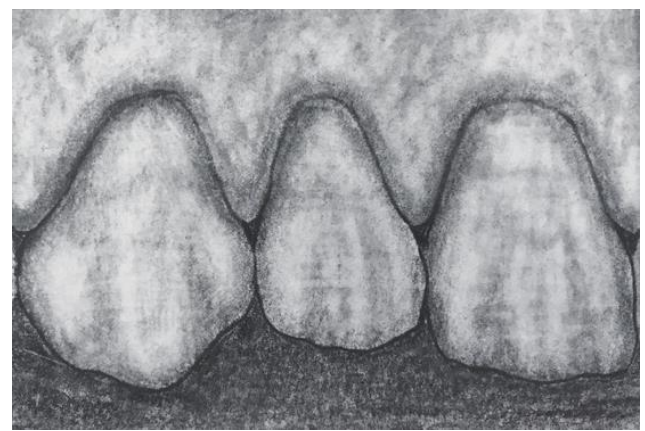

Figure 4. Class III. Lateral incisor in line with GAL

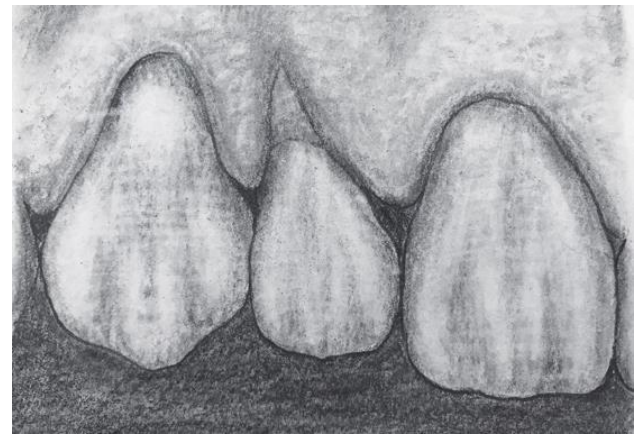

Figure 5. Class IV. Symmetry of gingival architecture or the architecture that cannot be assigned to the above groups. This occurs rather frequently due to periodontal disease, recessions or eccentric tooth position

Dental professionals are constantly exposed to the concept of an aesthetic smile through their experience and training. This level of exposure is likely to be much higher than for lay people. Studies have demonstrated that dentists are conditioned to have an overly critical view for any deviation from what is considered to be a "gold standard" (16). With media focusing on white pearly teeth and voluptuous lips with little regard given to gingival tissues, patients might have little awareness of "pink aesthetics" concepts. This in turn can result in dentists being more prone to aesthetic "overtreatment" while patients have less perceived need for ideal gingival display (17). Such differences may result in miscommunication when setting aesthetic treatment goals.

The aim of the study was to focus on the gingival components of smile design, comparing the differences in aesthetic preference between dental professionals and lay people which may be used to facilitate their aesthetic communication and treatment planning.

\section{MATERIALS AND METHODOLOGY}

The research investigated whether variations of maxillary gingival display influence perceived smile aesthetics. It also measured the difference of aesthetic preference between lay people and dental professionals as well as between general dental practitioners (GDPs) and orthodontic specialists. GDPs represent the largest group of dental professionals who are involved in the treatment and management of a patient's smile who have had training in smile aesthetics. Orthodontic specialists represent the group of dental professionals with the highest level of training in the topic of teeth arrangements and perceived aesthetics. Lay people were chosen 
as they represent patients, the recipients of such treatment or intervention from the dental professionals.

This study was based on the analysis of quantitative data, collected via a questionnaire which consisted of two sets of digitally altered photographs. One of the authors has copyright of the two original images. These digitally altered images were used as a tool to depict the smiles with different variations of gingival exposure. The variables allowed the respondents to rate the smiles from the most to the least attractive when viewed under standardised conditions. Using ranking order scale to measure aesthetic preference enabled the comparison between groups and individuals.

For the purpose of reducing the number of potential compounding variables, anterior smile view and retracted view photographs were digitally altered using digital software (Photoshop ${ }^{\circledR}$ CC 2018, Adobe Systems, San Jose, California). The goal of editing was to produce standardised images of an aesthetic smile close to ideal parameters (Figures 6-9).

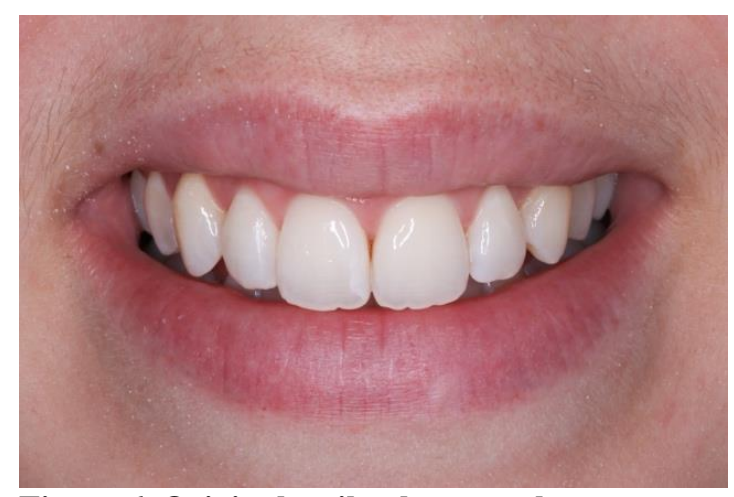

Figure 6. Original smile photograph

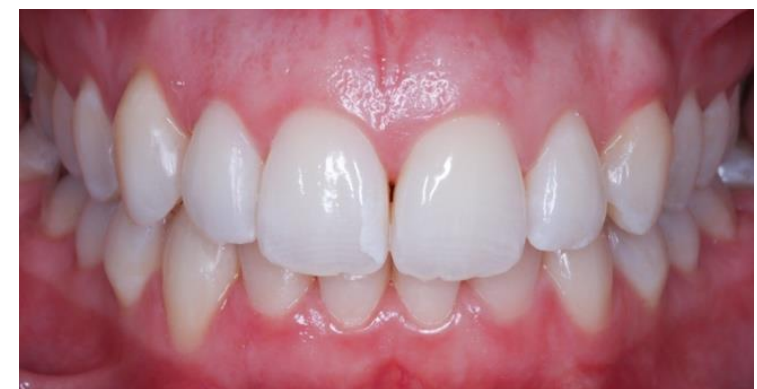

Figure 8. Original anterior retracted photograph

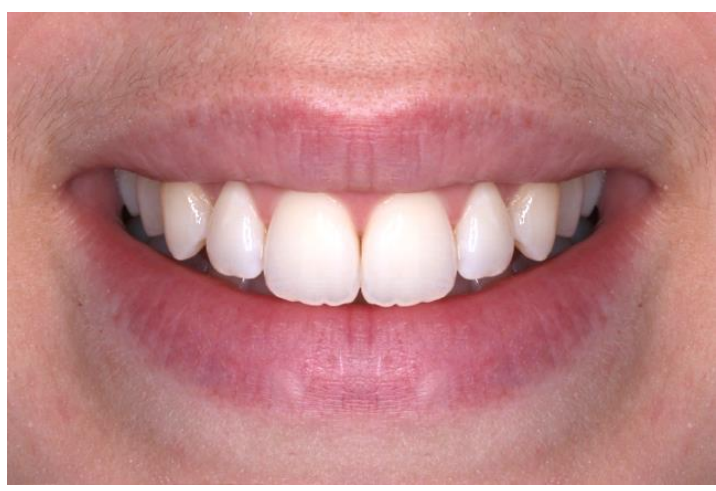

Figure 7. Altered smile photograph. (Symmetry of lips adjusted by mirroring left half of a smile).

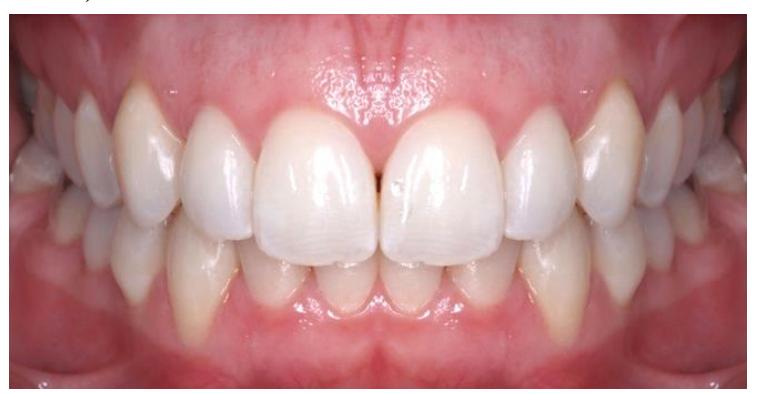

Figure 9. Altered anterior retracted photograph. (Symmetry of teeth and gingival tissues adjusted by mirroring left half of the arch).

These obtained images allowed further creation of variations of gingival display with minimal alterations of other smile components. Progressive increments of $3 \mathrm{~mm}$ were chosen to systematically move the lip superiorly or inferiorly to create a wider variation of gingival exposure occurring clinically (Figure 10). Five gingival amount variations were produced, and each of them could be classified either as low, average or high smile line (Figures 10-14). 


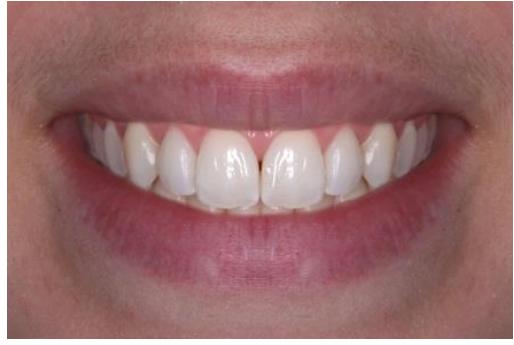

Figure 10. Average smile line (0).

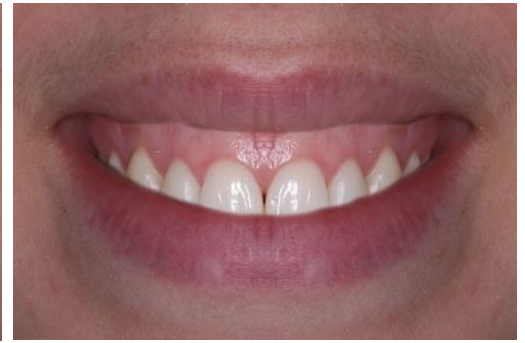

Figure 11. High smile line (-6)

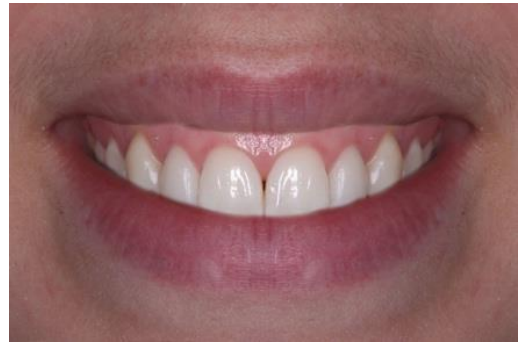

Figure 12. High smile line (-3)

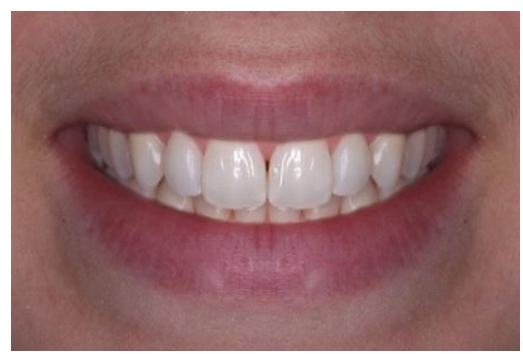

Figure 13. Average smile line (+3)

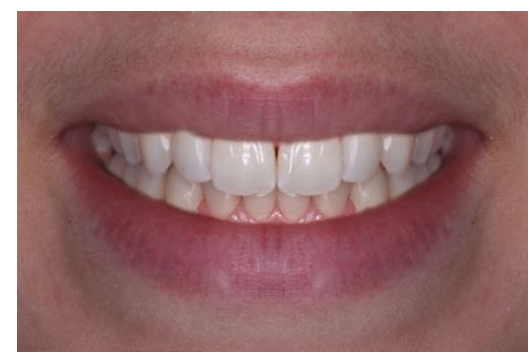

Figure 14. Low smile line (+6)

The second set of five images reflected the variations of gingival margin outline. Progression of gingival margin from central incisor to canine was digitally altered to create the architectures of Class I, II, III and symmetrical and asymmetrical Class IV gingival displays (Figures 1519).

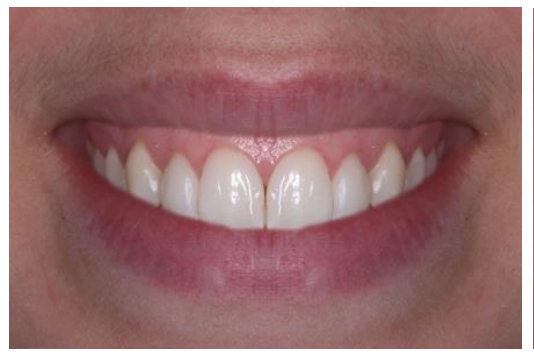

Figure 15 Class I gingival levels (C1) Figure 16. Class II gingival levels (C2)

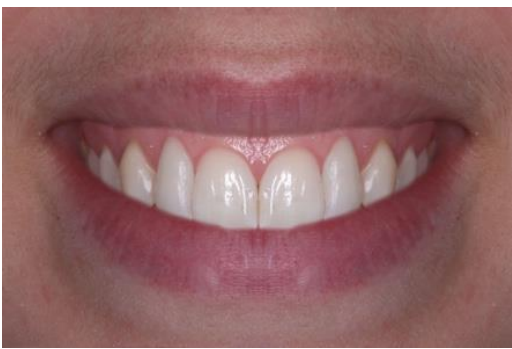

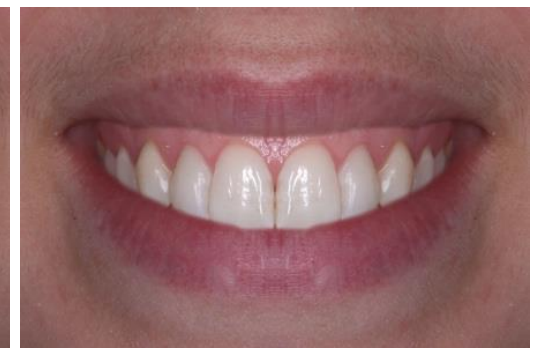

Figure 17. Class III gingival levels (C3)

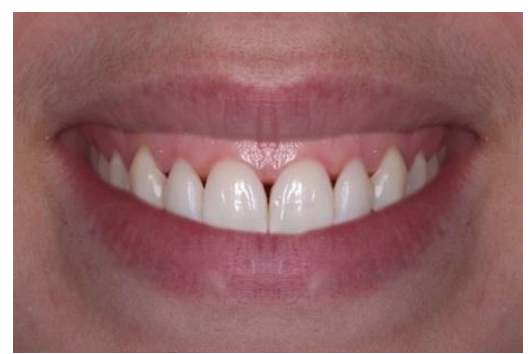

Figure 19. Symmetrical gingival levels with

\author{
black triangles (BT)
}

Figure 18. Asymmetrical gingival levels (AS)

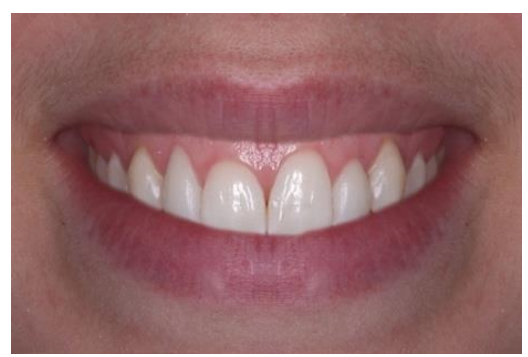


The images were printed on 300 gsm HP® Fujifilm ${ }^{\circledR}$ professional paper $\left(10 x 6 \mathrm{~cm}\right.$, Fujifilm ${ }^{\circledR}$, Tokyo, Japan) and were given identification codes visible to the researcher only (Tables 1,2). This very high quality paper was used in order to be able to print the best quality digital images for this study.

\begin{tabular}{|l|l|l|l|l|l|}
\hline $\begin{array}{l}\text { Amount of } \\
\text { gingival } \\
\text { display }\end{array}$ & & & werny & & \\
\hline Code & 0 & -6 & -3 & +3 & $\mathbf{+ 6}$ \\
\hline
\end{tabular}

Table 1. Set No1: codes for pictures with varying amount of gingival display

\begin{tabular}{|c|c|c|c|c|c|}
\hline $\begin{array}{l}\text { Architecture } \\
\text { of gingival } \\
\text { display }\end{array}$ & wonny & womply & vempy & worngy? & wrony \\
\hline Code & C1 & $\mathrm{C2}$ & C3 & $\mathbf{A S}$ & BT \\
\hline
\end{tabular}

Table 2. Set No2: codes for pictures with varying architecture of gingival display. $\mathrm{C} 1$ stands for Class 1 gingival architecture; C2 - Class II; C3 - Class III; AS - asymmetric Class IV; BT - black triangles Class IV.

A small pilot test was conducted to refine and finalise the research protocol for this study. This allowed the confirmation of preferred size of photographs, standardisation of the questionnaire and the integrity of the research protocol. The study was approved by UCL Research Ethics committee (Project ID Number): 6552/002.

\section{Research protocol}

Sample size calculations estimated that 124 respondents (60 lay people, 32 GDPs and 32 OS), were required to have $80 \%$ power to detect the difference to detect the difference between $60 \%$ preference and $30 \%$ preference at $5 \%$ level of significance $(18,19)$.

In order to reduce examiner bias, all participants were interviewed by the same researcher. Each participant was presented with a set of randomly shuffled images and asked to rank the images in order of attractiveness. The degree of agreement amongst repeated administrations of the test was tested by allowing a ten minute delay between initial and repeated rankings (20). During this delay, respondents were asked which smile component(s) (lips, teeth, dental or gingival components) were the most important when making their selections.

Anonymised data were recorded on an Excel spreadsheet and transferred to IBM ${ }^{\mathrm{R}}$ SPSS V.26 software package for statistical analysis. Pearson's chi-square test was used to investigate whether there were statistically significant differences between overall preferences for gingival aesthetics. Secondary analysis by logistic regression was used to determine if there were 
statistically significant preference differences between dental professionals and lay people. The influence of confounding factors to rankings was assessed by multivariate logistic regression.

\section{RESULTS}

Weighted Cohen's Kappa at the associated 95\% confidence intervals were calculated to assess the agreement between initial and repeated rankings. The results showed excellent global agreement between rankings both of the amount $(\kappa=0.906,95 \% \mathrm{CI})$ and the architecture $(\kappa=0.827,95 \% \mathrm{CI})$ of gingival display.

124 participants took part in the study (60 lay people [48\%]; 32 GDPs [26\%] and 32 orthodontic specialists [26\%]). Slightly more male dental professionals participated in the study whereas twice as many female patients volunteered (Table 1). Analysis of data collected from all three groups revealed preferences for the most and the least attractive gingival amount and architecture displays.

\begin{tabular}{|l|l|l|l|l|l|l|l|l|}
\hline & \multicolumn{2}{l}{ GDPs } & \multicolumn{2}{l|}{ Orthodontists } & \multicolumn{2}{l|}{ Lay People } & \multicolumn{2}{l|}{ Overall } \\
\hline & N & \% & N & \% & N & \% & N & $\%$ \\
\hline Male & 20 & 63 & 19 & 59 & 20 & 33 & 59 & 48 \\
\hline Female & 12 & 37 & 13 & 41 & 40 & 67 & 65 & 52 \\
\hline Total & 32 & $100 \%$ & 32 & $100 \%$ & 60 & $100 \%$ & 124 & $100 \%$ \\
\hline
\end{tabular}

Table 3. Gender distribution between groups.

\section{Preference for amount of gingival display}

All respondents perceived average smile with upper lip tangent to gingival margins as the most attractive (53.2\%). However, statistical analysis showed that the preference was on the borderline of statistical significance $\left(\chi^{2}(4)=144.15, \mathrm{p}=0.045\right)$. As for the least attractive amount, EGD with $6 \mathrm{~mm}$ of gingivae exposed received a clear majority of the votes amongst all three groups $(88.7 \%)$ and was found to be statistically significant $\left(\chi^{2}(4)=359.37, p=0.002\right)$.

When investigating the gingival amount preference amongst the groups, no statistically significant difference was found between dental professionals and lay $\left(\chi^{2}(4)=1.227, p=0.268\right)$. However, the perceived difference between GDP's and orthodontists was statistically significant $\left(\chi^{2}(4)=5.03, p=0.025\right)$. Investigation of the least attractive preferences amongst groups showed that there was no statistically significant difference in perception neither between dental professionals and lay people $\left(\chi^{2}(4)=0.00, \mathrm{p}=0.998\right)$, nor between GDP's and orthodontists $\left(\chi^{2}(4)=0.00, p=0.998\right)$. 


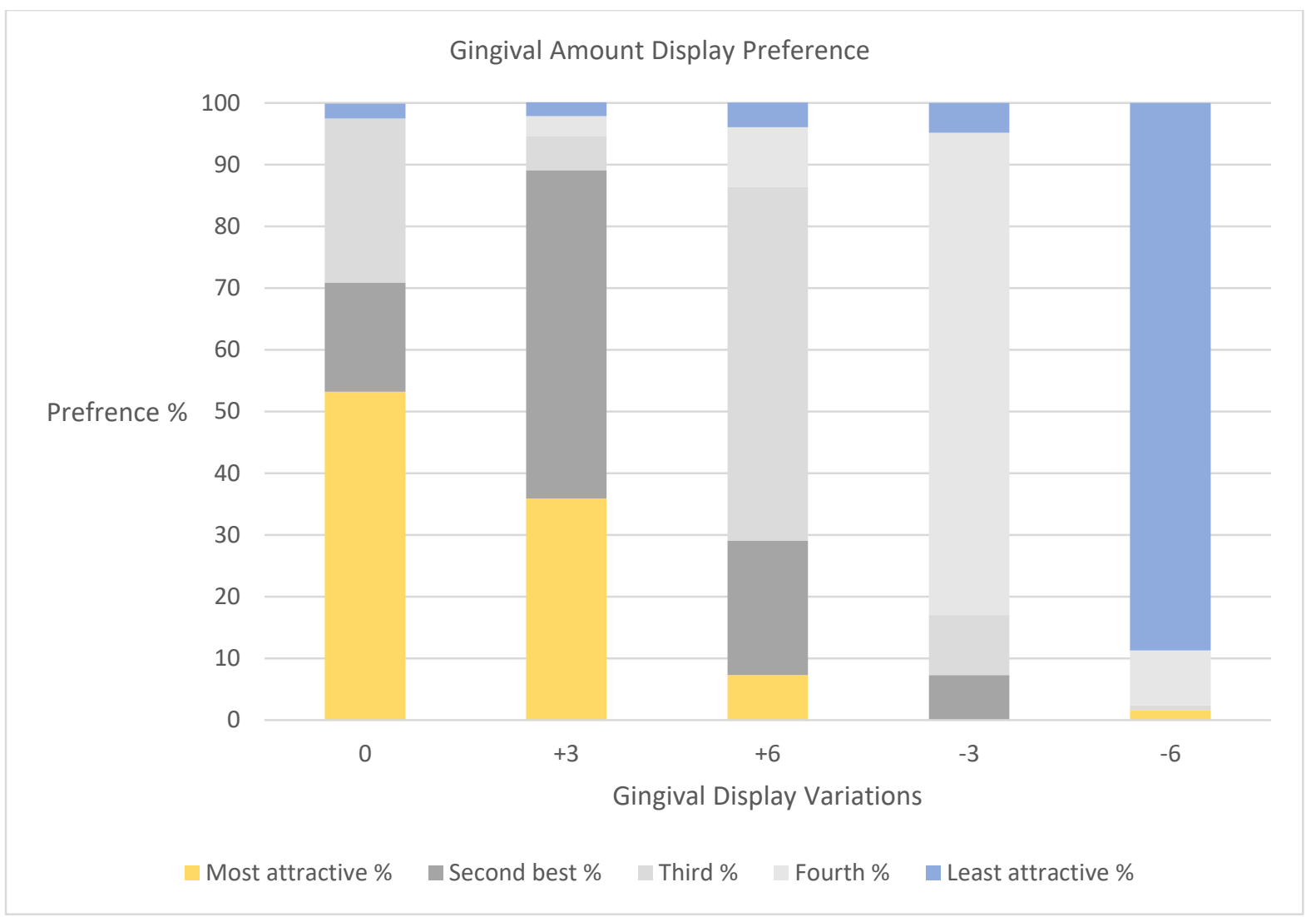

Figure 20. The ranking order of gingival amount display.

Analysis of components playing a role in the most attractive smile perception showed that all smile components including lips, teeth, dental and gingival components were equally important (60\%). Meanwhile, gingival component was the most influential when selecting the least attractive smile (44\%) (Figures 22, 23). Orthodontists were found to be particularly inclined to consider all components when selecting the most or the least attractive gingival amount $(97 \%)$. Lay people were found to be the only group that gave so much importance to teeth (40\%) when considering gingival amount aesthetics. 


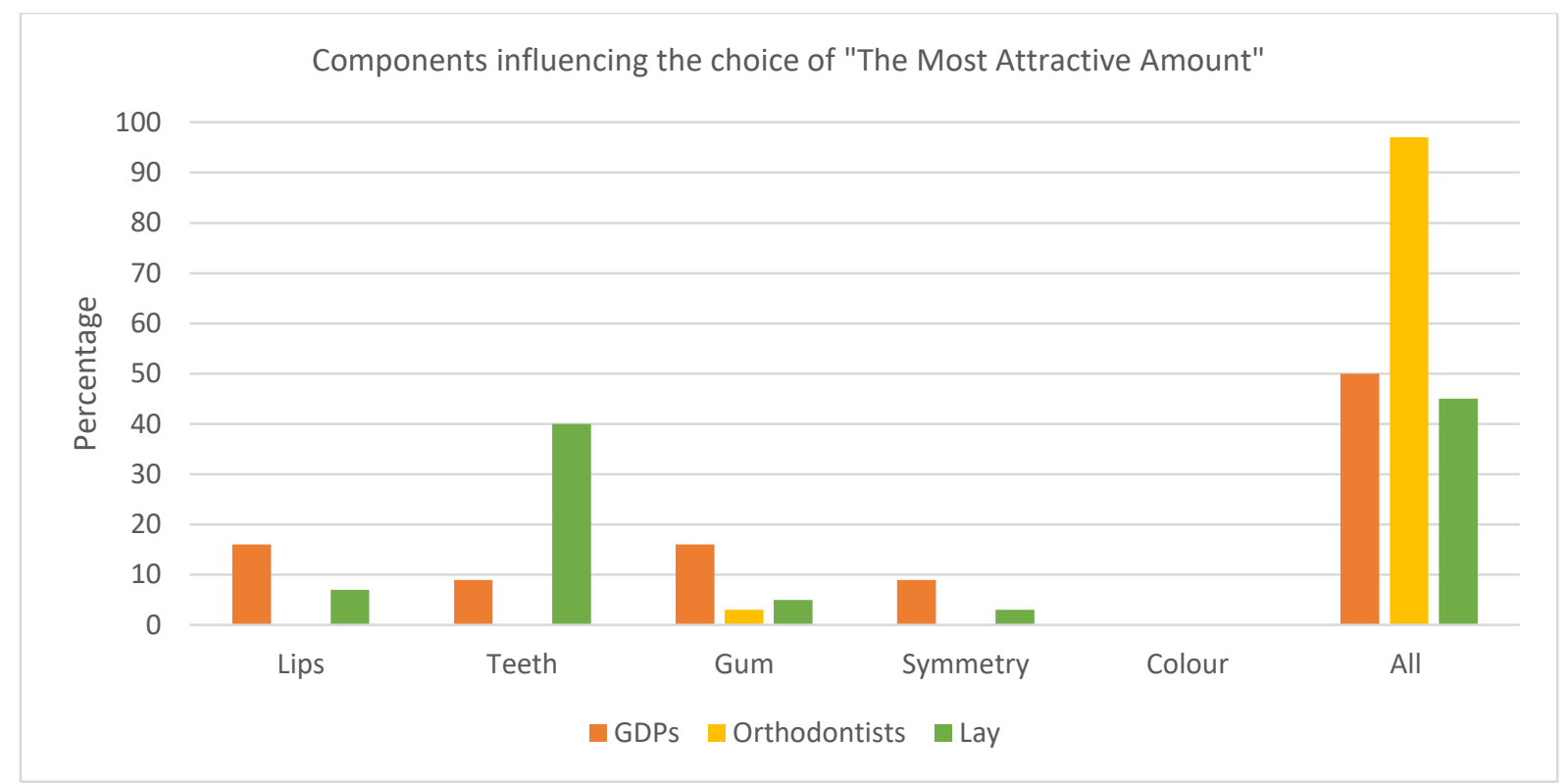

Figure 21. "The most attractive amount" influencing components.

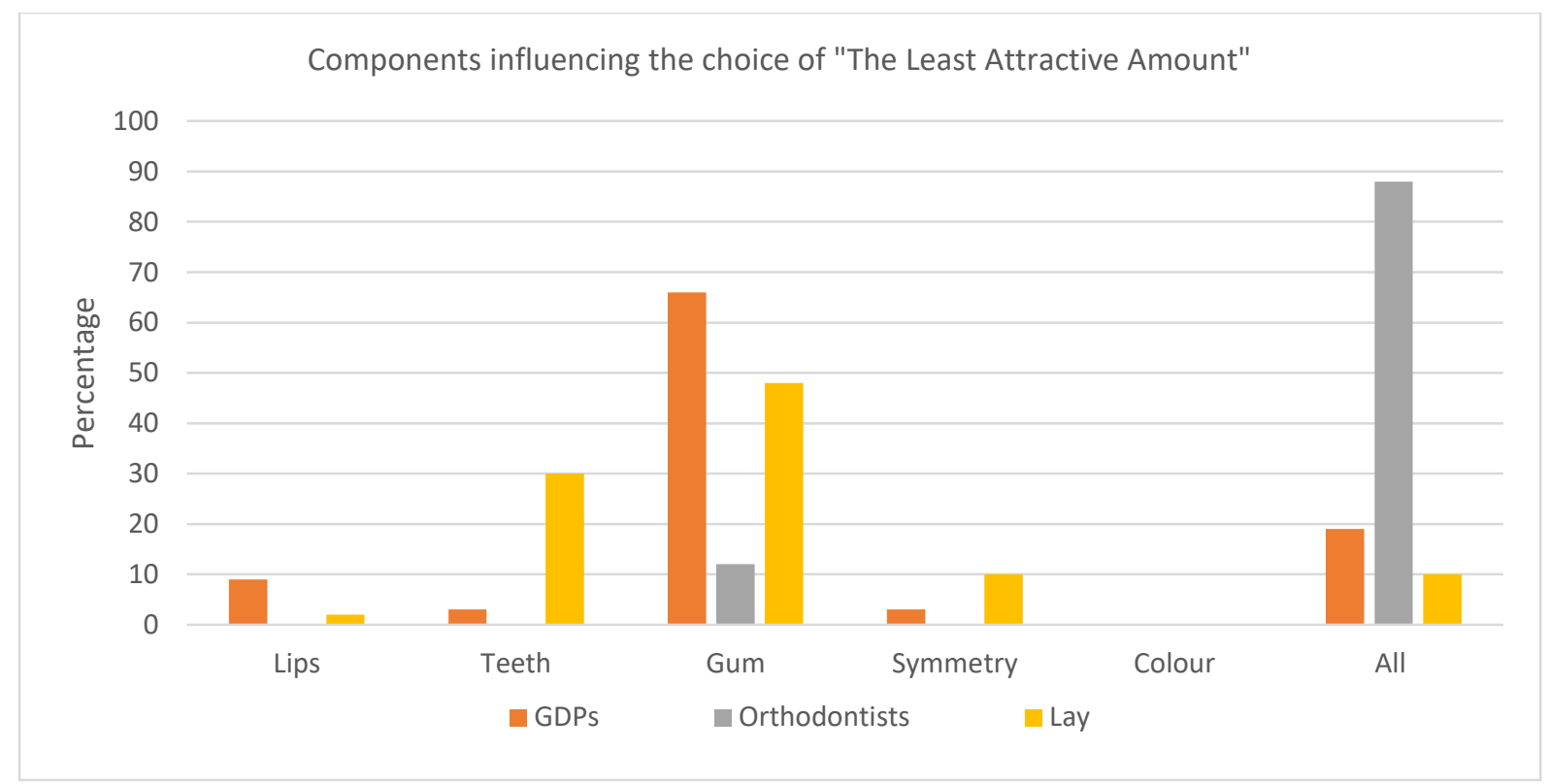

Figure 22. "The least attractive amount" influencing components.

\section{Preference for architecture of gingival display}

All the respondents perceived Class III gingival architecture as the most attractive $(65.3 \%)$ and this preference was found to be statistically significant $\left(\chi^{2}(4)=174.95, p=0.001\right)$ (Figure 21). Class IV gingival architecture with the loss of interdental papillae (black triangles) was recorded as the least attractive amongst all three groups (71.8\%). However, statistical analysis showed that this preference was not statistically significant $\left(\chi^{2}(4)=217.12, \mathrm{p}=0.192\right)$. 
Class III preference analysis between groups revealed statistically significant differences between dental professionals $\left(\chi^{2}(4)=10.87, \mathrm{p}=0.001\right)$ and lay people as well as between GDP's and orthodontists $\left(\chi^{2}(4)=7.33, \mathrm{p}=0.007\right)$. Dental professionals were more biased to select Class III gingival architecture as the most attractive when compared to lay people. Comparison of two dental professional groups showed that orthodontists were more biased towards Class III gingival architecture than GDP's. Investigation of the least attractive architecture preferences amongst groups showed that there was no statistically significant difference in perception between dental professionals and lay people $\left(\chi^{2}(4)=1.21, p=0.271\right)$, or between GDP's and orthodontists $\left(\chi^{2}(4)=0.00, \mathrm{p}=0.186\right)$.

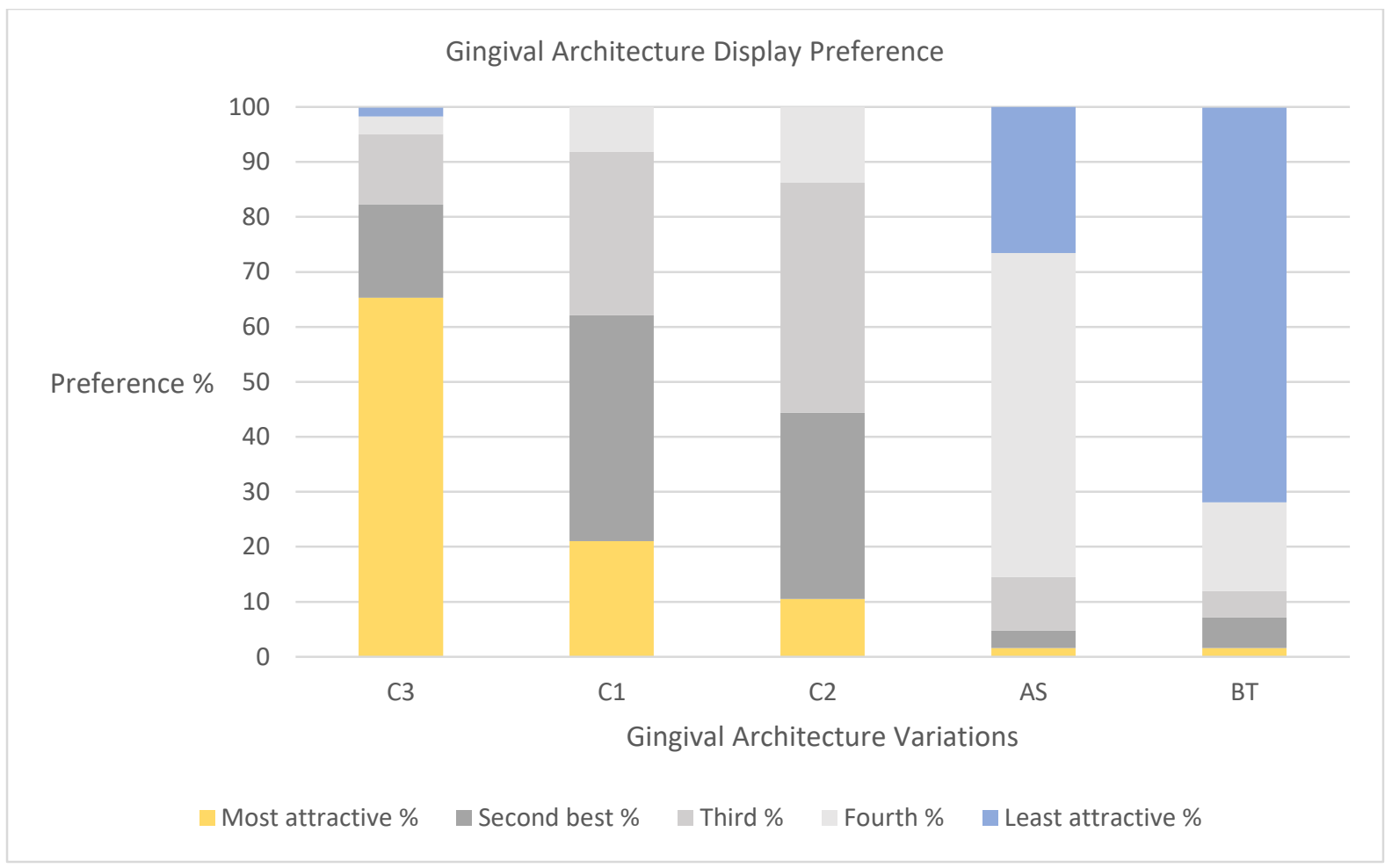

Figure 23. The ranking order of gingival architecture display.

Analysis of factors playing the most significant role in perceived gingival architecture revealed that all smile components are equally important when selecting the most attractive architecture of gingival display (Figures 25, 26). Orthodontists were found to be the most keen to consider all components (97\%), whereas GDPs and lay people also considered teeth (19\% and 27\% respectively), gingiva (19\% and 26\%) and symmetry (28\% and $22 \%$ ) to be as important as the combination of factors (34\% and 32\%). When selecting the least attractive architecture of gingival display, gingival components were the most influential (50\%). This was substantiated mostly by GDPs (63\%) and lay people (50\%). 


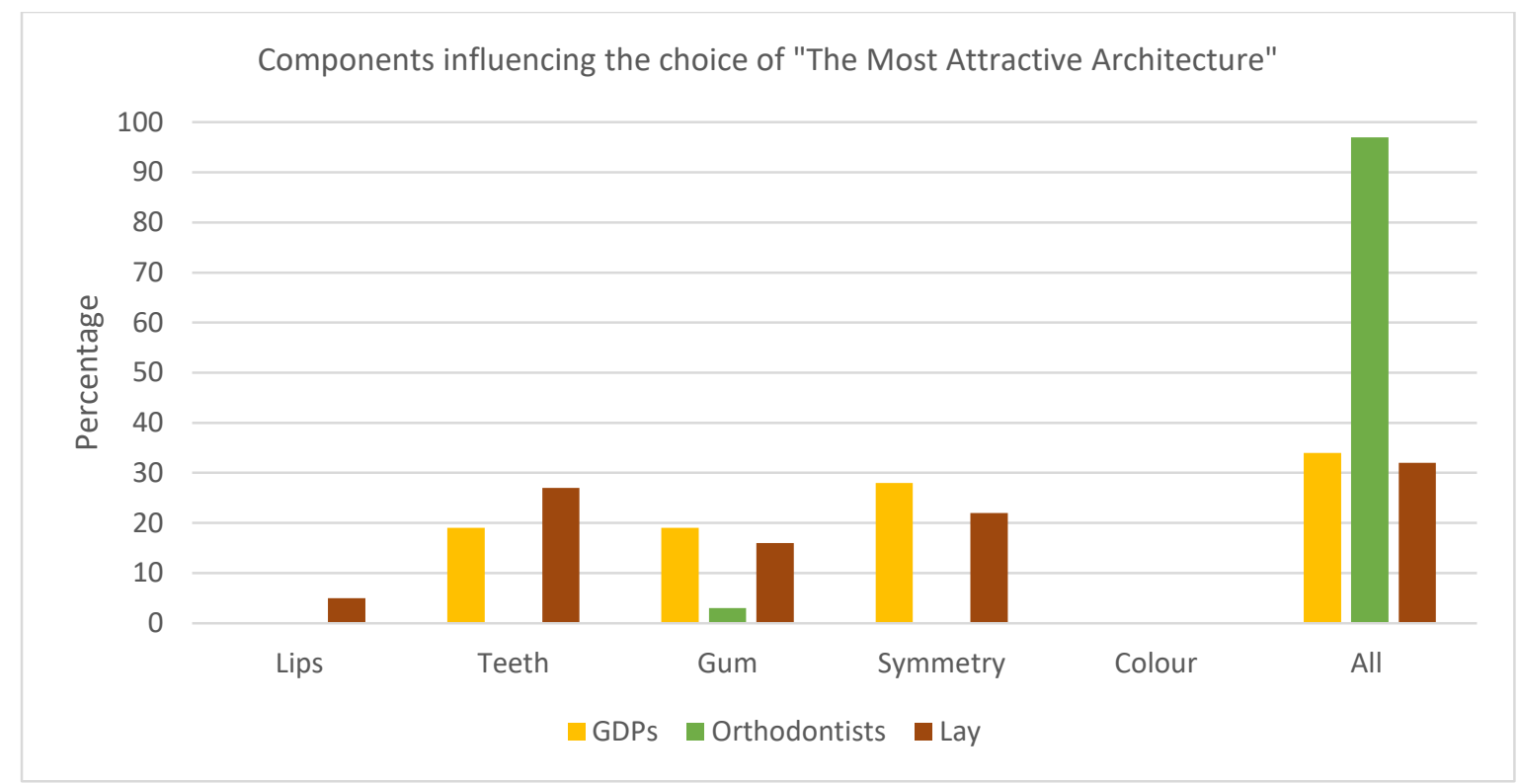

Figure 24. Influence of smile components for "The Most Attractive Architecture" choice.

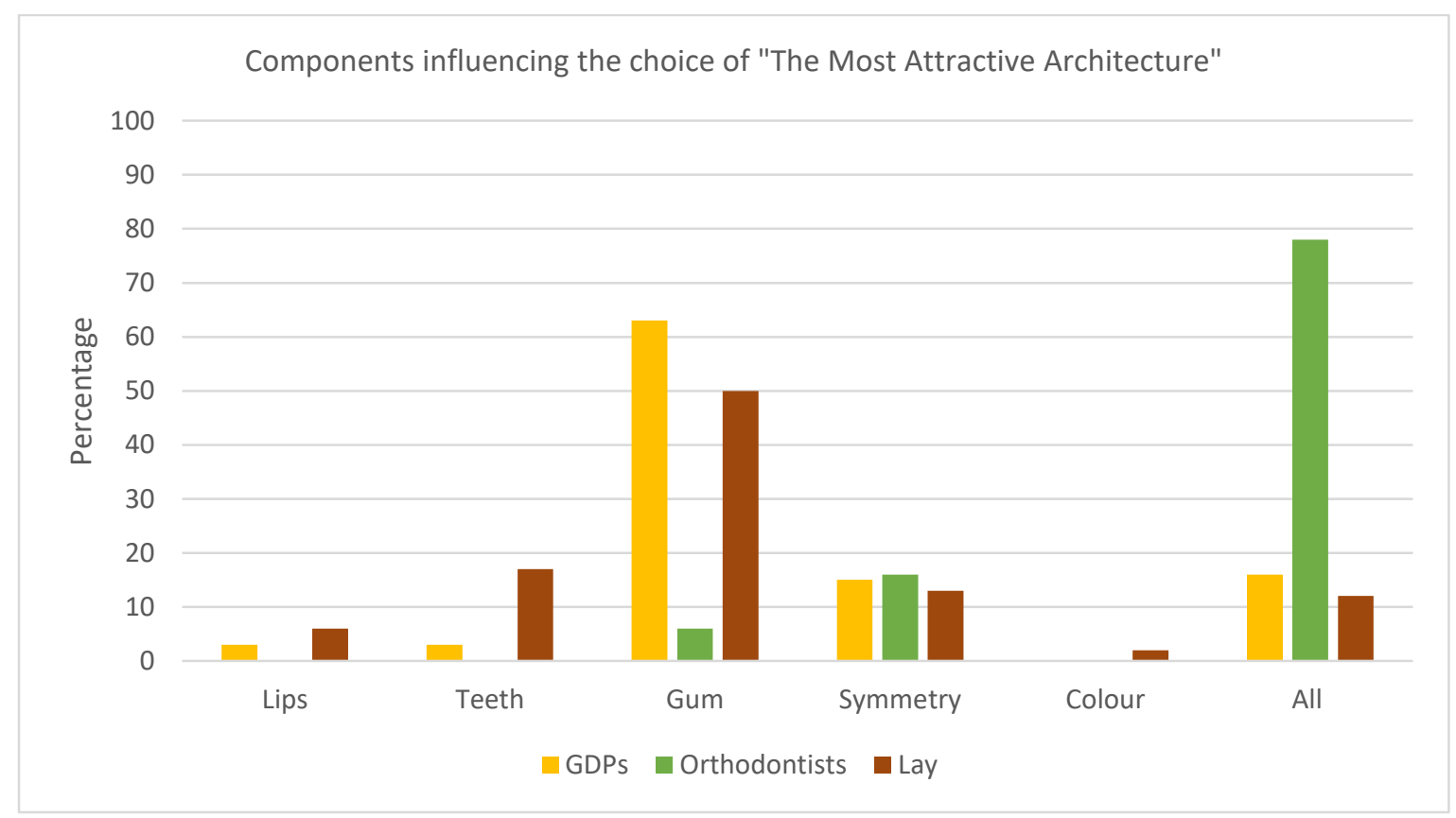

Figure 25. Influence of smile components for "The Least Attractive Architecture" choice. 


\section{DISCUSSION}

Conducting aesthetic research is challenging due to the variety of methods and protocols available. This research was conducted by the insider researchers whose in-depth knowledge and understanding of the topic allowed extraction of data in the most effective way whilst minimising the risk of introducing researcher's bias (21). To minimise potential bias, several strategies were included: i) strict adherence to research protocol, ii) piloting the study to assure relevant validity, iii) using standardised questionnaire for data generation and iv) collection and collation of data for analysis and discussion.

A picture of an ideal smile was required. Perfect smiles rarely occur in nature and therefore digital manipulations were performed on a set of photographs, where the authors have copyright, in order to alter the smile to a described ideal (22). In order to create variations in gingival display, lip position $(23,24)$, vertical teeth position $(25-27)$ or teeth proportion required appropriate adjustments. This in turn resulted in the introduction of a variable which was not the primary focus of this study. It should be noted in real life aesthetic dentistry, that even when a single variable is adjusted, the appearance of another variable is inevitably affected. In this paper, alteration of the amount of gingival display altered the position of the incisal edge and the relationship with the lower lip line. Furthermore, alterations of gingival architecture impacted on teeth proportions. As noted by Guo et al. (23), the results of the studies where multiple confounding factors are introduced do not always reflect completely the true outcomes although these are accepted limitations of these studies.

Conversely, although the position of the incisal edge in this study participated as a secondary variable, the overall appearance of the smile was maintained aesthetic and realistic with a primary focus evidently kept on gingival components. The difficulty to isolate a single variable supports the realism that no smile component is independent and only exists in relation to other smile components (28). Therefore, it can be suggested that organic and more perceptual ideal smile planning approach should be adopted. This is in line with a recent study by Pereira Silva et al. (29) who advocate intuitive "facial flow concept" in order to respect unique nature of each human face instead of mandatory adherence to static smile design principles.

While large sample sizes generate more accurate and practical meaning, to have the required statistical power to validate the data generated and thereby the results, the calculated minimum sample size of respondents for this research was 124, which was exceeded in this piece of work. The results therein are valid enough to interpret correlations between gingival display and smile aesthetics.

The data was drawn from arbitrary ranking of the aesthetic preference, therefore the results must be consistent in order for the study method to be reliable (30). Excellent scores of reliability measure indicate that the conclusions drawn from this study can be considered to be valid (19). Greater scores for the amount rather the architecture of gingival display signifies that respondents, lay people in particular, are more consistent when ranking the amount of gingival display. It can be speculated, that respondents are more perceptive to the changes of amount of gingival tissues showing when smiling. Meanwhile, the architecture of gingival 
margins is more subtle and lay people, who are not usually accustomed to paying such great attention to gingival aesthetics, find this more challenging to rate.

\section{Preference for the amount of gingival display}

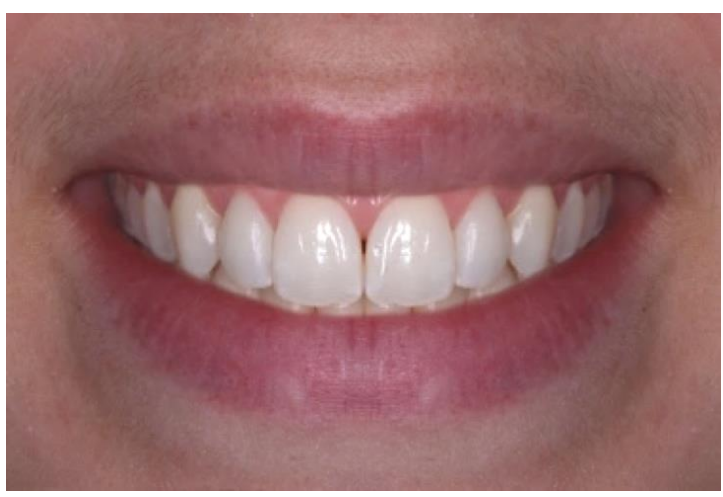

Figure 26. Average smile line

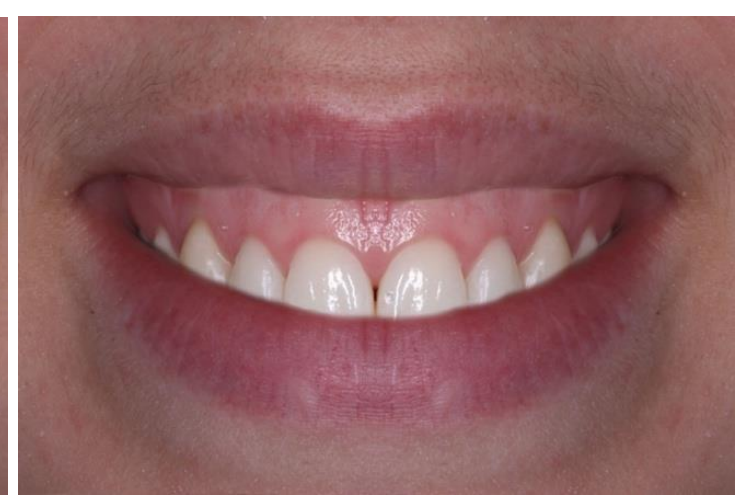

Figure 27. Excessive gingival display (EGD)

The findings of this study were that average smile line (Figure 26) would be the most attractive and excessive gingival display (Figure 27) would be the least attractive were in line with many other comparable studies $(24,31-36)$. However, the results of this study showed little evidence to suggest that the average smile line is the most preferred by the population. It therefore implies that a smile with ideal teeth and lip components can have variations in gingival display and yet still be perceived as attractive. Conversely, there was strong evidence that EGD can contributed to perceived aesthetic failure even if other smile components were ideal. Similar findings are reflected in the literature that reports wide variations of preference for amount of gingival display $(24,25,33,34,37,38)$. It should also be noted, that all studies differed greatly in methodologies and therefore the results cannot be considered comparable to determine universal threshold of acceptable gingival display.

There is little doubt that there is no universally accepted amount of gingival display, and participants are tolerant to variations as long as the exposure is not perceived to be extreme. In other words, different participants favour different amounts of gingival display, although most of them agree on what is deemed unattractive. Similar preferences are shared amongst dental professionals and lay participants. However, orthodontists seemed to have the strongest opinion about the attractiveness of the amount of gingival display. When the amount of gingival exposure is increased significantly, it becomes a negative focus of attention and is perceived as unattractive. Hence all focus groups of this study (dental professionals and lay people) consistently perceived excessive gingival display as unattractive.

\section{Preference for the architecture of gingival display}

The results of the gingival architecture analysis concluded that there was a general preference for symmetrically levelled Class III gingival architecture when significant amount of maxillary gingival tissues was exposed. This is contrary to a common philosophy that Class I gingival 
architecture is the most desirable $(12,13)$. However, lay respondents were more tolerant to variations whereas dental professionals, especially orthodontists, were keener to choose this type of gingival architecture as the most attractive. It should be noted that this study investigated architectural preference on smiles with excessive gingival display. In such cases, levelled gingival margins create a harmonious symmetry, reduce a negative impact of wide amount of gingival exposure and thus create a perception bias.

Furthermore, Class IV symmetrical black triangle architecture was disapproved similarly by both dental professionals and lay respondents. Although it was reported as being the least attractive, this study demonstrated that there was no evidence to claim that the loss of interdental papillae could be solely accountable for the aesthetic failure.

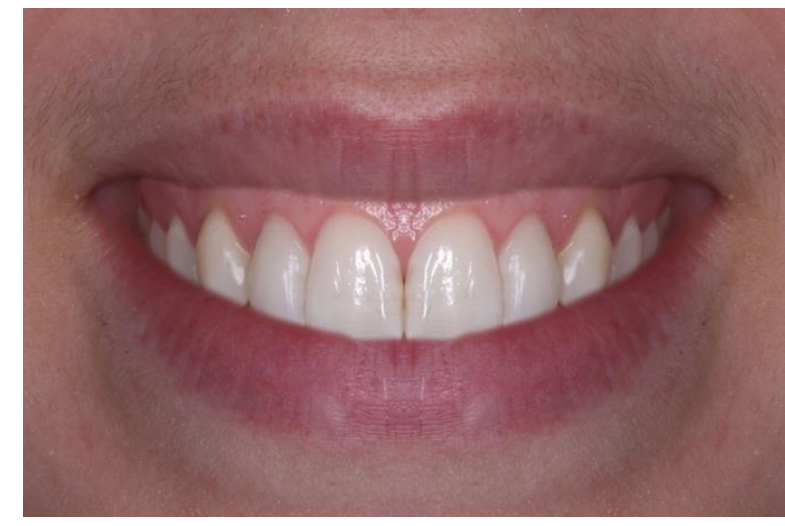

Figure 28. Class III gingival architecture triangles)

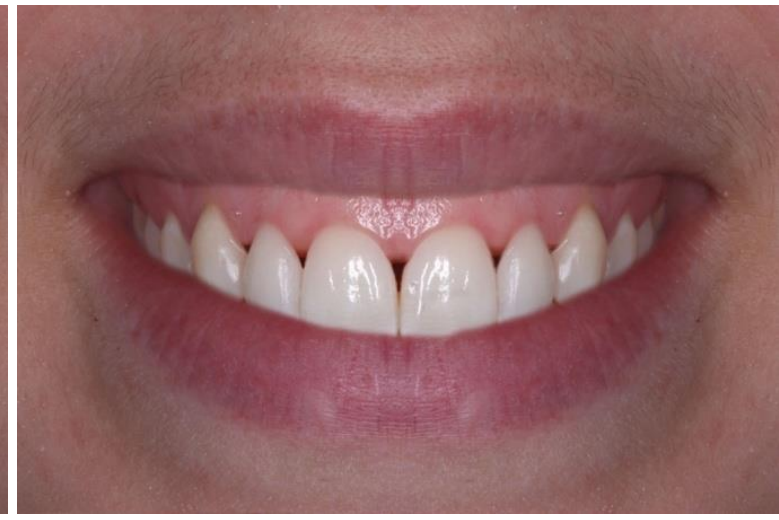

Figure 29. Class IV gingival architecture (black

Very few recent studies are available that exclusively measure the aesthetics of gingival architecture. Machado et al., (22) reported both Class I and Class III were attractive while Ker et al. (27) and Kokich et at., (31) reported there was no preference for gingival architecture attractiveness. Witt and Flores-Mir (36) concluded that gingival architecture preference was dependent on the configuration of teeth. It should be noted that most of the studies that used altered gingival architectures also displayed full length of maxillary teeth and the contour of the incisal edges. When gingival levels are altered, there is an inevitable change either in the proportions of the teeth or/and the configuration of the incisal edges. Therefore, it introduces more perceptible dental variables that can be accounted for aesthetic perception which might suggest erroneous conclusions. This study used high smile line without incisal configuration visible. However, the change of teeth proportion was inevitable and therefore the results about gingival architecture should be considered with caution.

\section{Factors influencing aesthetic preference}

Analysis of how respondents were making the decisions about their aesthetic preference revealed some interesting common trends. 
When selecting the most aesthetic gingival display, the respondents considered all smile components, including lips, teeth, gingiva, symmetry and colour. This implies that aesthetic smile perception is multifactorial, and that one single component cannot be accounted for an overall aesthetic result. This supports the macro-aesthetic theory described by Rifkin and McLaren (5) and Morley and Eubank (39), who state that the whole visual effect of all components is greater than their sum.

However, when selecting the least attractive gingival display, a single component (typically the most extreme) was found to be the most influential in aesthetic perception. In this study, respondents picked "gum" or "symmetry" as the most important factors when making the decision about poor aesthetics. With the exception of the orthodontist respondents, all other groups identified the most extreme factor disturbing perceptual coherence the most. In the meantime, orthodontists tended to consider all the variables together "as a whole" without focusing on any particular smile component.

Furthermore, analysis of how different groups substantiate their aesthetic choices illustrated that visual perception of GDP's and lay respondents was similar. Both groups inclined to select the same influential factors at the same importance rate. Meanwhile, orthodontists not only were the most keen to adhere to "gold standard" aesthetic smile concepts, they also considered all smile components as being equally influential when selecting the most or the least attractive perceived smile. Perhaps due to the nature of their work, orthodontists are the most aware of smile design concepts amongst the respondents, thus creating such tendencies. Lay respondents, on the other hand, paid more attention to teeth than dental professionals when considering smile aesthetics. This reflects that lay people are typically more conscious about the appearance of the teeth, and might have relatively little awareness about other the smile components.

\section{CONCLUSION}

Within the limitations of this study, a few conclusions could be drawn about the role of gingival display in perceived smile aesthetics.

Perceptions are notably stronger regarding specific gingival display noted as being unattractive rather than attractive. In other words, respondents are relatively open-minded about variations in gingival display as long as they are not extreme. This allows the trained dental professionals a more organic approach when designing an "acceptable" smile. In other words, it affords the dental professional the clinical freedom to customize the height of the smile satisfactorily by selecting from a range of aesthetic variations instead of just adhering to a static number.

Class III gingival architecture is the most preferred gingival architecture type when excessive amount of maxillary gingiva is exposed while smiling. Such levelled and symmetrical gingival margins reduce negative visual impact of excessive gingival display and thus can be a useful tool for the dental professionals when treating patients with a high smile line. Although smiles with "black triangles" are disliked the most by the respondents, it is unlikely to be the only gingival archetype to lead to aesthetic failure. 
Lay respondents demonstrated more tolerance for wider variations of what was considered to be aesthetic when compared to the dental professional respondents. However, GDPs and lay respondents shared very similar aesthetic views. Orthodontist respondents were the most strict and critical in adhering to the golden rules of aesthetic gingival display when compared to GDPs and lay respondents. These differences in perceptions highlight the importance of effective communication between patients and dental professionals when setting aesthetic treatment goals. Dental professionals, should involve patients into treatment planning at all times.

When considering attractiveness of the smile, the combination of all smile components (lips, teeth, gingiva) would be taken into consideration. However, when unattractiveness is perceived, the most deviant single component would became the centre of focus and dissent. When this happens, lay respondents were more likely to focus on teeth than any other smile component. It is therefore very important for dental professionals to educate the patients the fact that all smile components are equally important in order to achieve an optimum comprehensive aesthetic outcome. This highlights the fact that aesthetic treatment is more than white pearly teeth which often requires a complex and interdisciplinary approach.

Further research to replicate the results in a larger scale will be very desirable. It will further ameliorate any potential limitations of this study by adopting more comprehensive mixed data method approaches.

\section{Declaration of Competing Interest}

The authors declare that they have no known competing financial interests or personal relationships that could have appeared to influence the work reported in this paper.

\section{Acknowledgements}

This research did not receive any specific grant from funding agencies in the public, commercial, or not-for-profit sectors. 


\section{References:}

1. Eagly AH, Ashmore RD, Makhijani MG, Longo LC. What Is Beautiful Is Good, But...: A MetaAnalytic Review of Research on the Physical Attractiveness Stereotype. Psychological Bulletin. 1991;110(1):109-28.

2. Livingstone M, Hubel D. Segregation of Form, Color, Movement, and Depth: Anatomy, Physiology, and Perception. Science. 1988;240(4853):740.

3. Giddon DB. Orthodontic applications of ps chologicaland perceptual studies of facial esthetics. Seminars in Orthodontics. 1995;1(2):82-93.

4. Epstein D, Herzberger B, Rentschler I. Beauty and the brain : biological aspects of aesthetics / edited by Ingo Rentschler, Barbara Herzberger and David Epstein. Basel: Basel : Birkhauser; 1988.

5. Rifkin R, McLaren E. Macroaesthetics: Facial and Dentofacial Analysis. Oral Health. 2005;95(11):65-77.

6. Sharma PK, Sharma P. Dental Smile Esthetics: The assessment and Creation of the Ideal Smile. Seminars in Orthodontics. 2012;18(3 (September)):193-201.

7. Tjan AHL, Miller GD, The JGP. Some esthetic factors in a smile. The Journal of Prosthetic Dentistry. 1984;51(1):24-8.

8. Allen E. Use of mucogingival surgical procedures to enhance esthetics. Dent Clin North Am. 1988(32):307-30.

9. Vig RG, Brundo GC. The kinetics of anterior tooth display. The Journal of Prosthetic Dentistry. 1978;39(5):502-4.

10. Peck S, Peck L, Kataja M. Some vertical lineaments of lip position. American Journal of Orthodontics \& Dentofacial Orthopedics. 1992;101(6):519-24.

11. Ahmad I. Anterior dental aesthetics: Gingival perspective. British Dental Journal. 2005;199(4):195.

12. Rufenacht CR. Fundamentals of esthetics Chicago London: Quintessence Pub. Co.; 1990.

13. Lindhe J. Textbook of clinical periodontology. Seibert J, Lindhe J, editors. Copenhagen, Munskagaard1989.

14. Duran GS, Dindaroglu F, Gorgulu S. Three-dimensional evaluation of social smile symmetry. Angle Orthod. 2017;87(1):96-103.

15. Sarver DM, Yanosky M. Principles of cosmetic dentistry in orthodontics: Part 2. Soft tissue laser technology and cosmetic gingival contouring. American Journal of Orthodontics \& Dentofacial Orthopedics. 2005;127(1):85-90.

16. Shaw WC, H. G. Lewis, and N. R. E. Robertson. Perception of malocclusion Br. Dent. J. 138 : 211, 1975. British Dental Journal; 1977. p. 211-6.

17. Goldstein RE. Attitudes and Problems Faced by Both Patients and Dentists in Esthetic Dentistry Today: An AAED Membership Survey. Journal of Esthetic and Restorative Dentistry. 2007;19(3):164-70.

18. Casagrande JT, Pike MC. An improved approximate formula for calculating sample sizes for comparing two binomial distributions. Biometrics. 1978;34(3):483.

19. Bujang MA, Baharum N. Guidelines of the minimum sample size requirements for Cohen's Kappa. Epidemiology Biostatistics and Public Health. 2017;14(2):e12267-1-e-10.

20. Bornstein RF. Exposure and Affect: Overview and Meta-Analysis of Research, 1968-1987. Psychological Bulletin. 1989;106(2):265-89.

21. Merton RK. Insiders and Outsiders: A Chapter in the Sociology of Knowledge. American Journal of Sociology. 1972;78(1):9-47.

22. Machado AW. 10 commandments of smile esthetics. Dental Press Journal of Orthodontics. 2014;19(4):136-57. 
23. Guo J, Gong H, Tian W, Tang W, Bai D. Alteration of gingival exposure and its aesthetic effect. The Journal of craniofacial surgery. 2011;22(3):909.

24. Kokich V, Kokich V, Kiyak H. Perceptions of dental professionals and laypersons to altered dental esthetics: Asymmetric and symmetric situations. Am J Orthod Dentofac Orthop.

2006;130(2):141-51.

25. Ioi H, Nakata S, Counts AL. Influence of gingival display on smile aesthetics in Japanese. Eur J Orthod. 2010;32(6):633-7.

26. Kaya B, Uyar R. Influence on smile attractiveness of the smile arc in conjunction with gingival display. American Journal of Orthodontics \& Dentofacial Orthopedics. 2013;144(4):541-7.

27. Ker AJ, Chan R, Fields HW, Beck M, Rosenstiel S. Esthetics and smile characteristics from the layperson's perspective: A computer-based survey study. J Am Dent Assoc. 2008;139(10):1318-27.

28. Ahmad I. Anterior dental aesthetics: Dentofacial perspective. British Dental Journal. 2005;199:81.

29. Pereira Silva BM, Eduardo; Stanley, Kyle; Coachman, Christian. The facial flow concept: An organic orofacial analysis of the vertical component. The Journal of Prosthetic Dentistry. 2019;121(2):189-94.

30. Woolson RF. Statistical methods for the analysis of biomedical data / Robert F. Woolson, William R. Clarke. 2nd ed. ed. Clarke WR, editor. New York: New York : Wiley-Interscience; 2002. 31. Kokich VO, Asuman Kiyak H, Shapiro PA. Comparing the Perception of Dentists and Lay People to Altered Dental Esthetics. Journal of Esthetic and Restorative Dentistry. 1999;11(6):311-24. 32. Feu D, Fabíola Bof de A, Ana Paula Camata N, José Augusto Mendes M, Antonio Augusto G, Jonas Capelli J. Percepção das alterações no plano gengival na estética do sorriso Perception of changes in the gingival plane affecting smile aesthetics. Dental Press Journal of Orthodontics. 2011;16(1):68-74.

33. Parrini S, Rossini G, Castroflorio T, Fortini A, Deregibus A, Debernardi C. Laypeople's perceptions of frontal smile esthetics: A systematic review. American Journal of Orthodontics \& Dentofacial Orthopedics. 2016;150(5):740-50.

34. Pausch NCK, Dimitrios. Gender Specific evaluation of variation of maxillary exposure when smiling. Journal of Carni-Maxillo-Facial Surgery. 2017;45:913-20.

35. Suzuki L, Machado AWL, Bittencourt MAV. Perceptions of gingival display aesthetics among orthodontists, maxillofacial surgeons and laypersons = Estudo comparativo da percepção estética do sorriso gengival por ortodontistas, cirurgiões buco-maxilo-faciais e leigos. Revista Odonto Ciência. 2009;24(4):367-71.

36. Witt M, Flores-Mir C. Laypeople's preferences regarding frontal dentofacial esthetics Periodontal factors. J Am Dent Assoc. 2011;142(8):925-37.

37. Ker AJ, Chan R, Fields HW, Beck M, Rosenstiel S. Esthetics and Smile Characteristics From the Layperson's Perspective: A Computer-Based Survey Study: A Computer-Based Survey Study. The Journal of the American Dental Association. 2008;139(10):1318-27.

38. Pithon MM, Santos AM, Campos MS, Couto FS, dos Santos AF, Coqueiro RdS, et al. Perception of laypersons and dental professionals and students as regards the aesthetic impact of gingival plastic surgery. European Journal of Orthodontics. 2014;36(2):173-8.

39. Morley J, Eubank J. Macroesthetic elements of smile design. The Journal of the American Dental Association. 2001;132(1):39-45. 